\title{
Safety Voltage Control Strategy for High Voltage Ride-Through of Doubly Fed Induction Generator (DFIG)
}

\author{
He Zehua \\ Mechanical and Electrical Engineering College,Hainan University Haikou city of Hainan Province China \\ hezehua_hn@163.com
}

Key words: high voltage ride-through; DFIG; safety voltage; DC-side voltage control

\begin{abstract}
DFIG high voltage ride-through method with the consideration for the safe operation voltage of the generator unit is proposed in the article. Based on the dynamic reactive support capability of DFIG, during grid voltage swell, the reactive powers outputted by the grid-side converter and the generator stator are concertedly controlled so as to keep the safe and stable operation of DC-side bus voltage. Then, the generator stator-side reactive output capability is preferentially considered to provide the reactive power that shall be outputted by the grid-side converter and the stator of DFIG while ensuring the safe port voltage. Finally, the formula for the operation range of the safety voltage is proposed according to the operation range of the grid-side converter and DFIG, thus to provide the basis for checking whether the internal components of DFIG can normally operate during the high voltage ride-through thereof.
\end{abstract}

\section{Introduction}

Along with the rapid development of wind power utilization, the guide rule, test requirement and adaption specification for introducing wind power generation to power grid have been successively proposed in some countries, and accordingly higher and higher requirement has been proposed for the fault ride-through capability of wind turbine generators. At present, the researches at home and abroad on the low voltage fault ride-through capability have gradually become mature. Although various wind power generation enterprises in China have different technical routes, they still have basically realized the low voltage ride-through capability in recent two years. However, for the grid disconnection of wind power generation usually caused by low voltage due to short circuit, the duration of the intermediate and low voltage is usually very short (only dozens of millisecond) in practical operation. Usually, the high voltage resulted from reactive overcompensation finally causes the shutdown of the wind power generators in the wind power plant. Therefore, people pay more and more attention to high voltage ride-through (HVRT). Internationally, the power grid regulations of such countries as Australia, U.S., Germany, Spain and Denmark have preliminarily standardized HVRT of wind power generators. For example, it is required in Australian power grid regulation that the wind power generators shall not have the disconnected operation for $60 \mathrm{~ms}$ when the grid voltage at the interconnection point swells to 1.3 times of the nominal voltage. Relevant HVRT requirements and test standards have not yet been proposed for the wind power generation enterprises in China, because HVRT is proposed as a new issue in recent two years and relevant researches are currently lacked in China.

\section{Reactive power constraint of DFIG during grid voltage swell}

The working principle of DFIG is as shown in Fig. 1. Under normal operation, active and reactive power decoupling control is adopted for rotor-side converter (RSC) and grid-side converter (GSC) in order to adjust the active and reactive powers outputted by DFIG stator through controlling the exciting current outputted by RSC, wherein GSC control aims at stabilizing DC-side voltage. When DC capacitor voltage is higher than the maximum continuous operating voltage, the off-load Chopper circuit in parallel connection in DC bus can be triggered for break-over to restrain the possible bus voltage pumping-up at the moment of sudden voltage change so as to ensure the safety of DC link. 


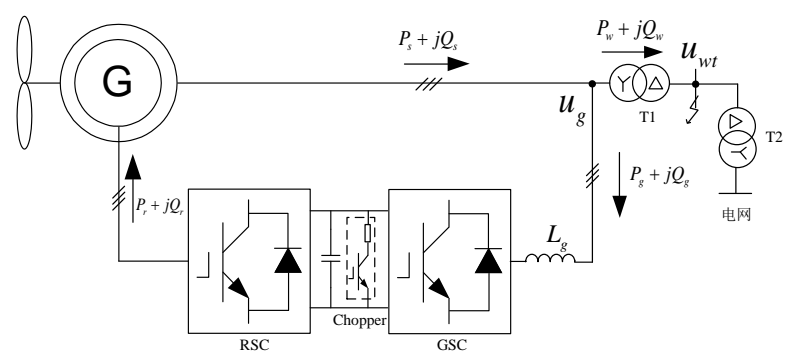

Fig1. The Structure of DFIG

In Fig. $1, u_{\mathrm{g}}$ is the voltage at the access point of GSC, $u_{\mathrm{wt}}$ is the grid-side voltage of the transformer at wind power generator port, $\mathrm{P}_{\mathrm{g}}$ is the active power outputted by GSC, $\mathrm{P}_{\mathrm{s}}$ is the active power outputted at the stator side of DFIG, $Q_{g}$ is the reactive power outputted by GSC, $Q_{s}$ is the reactive power outputted at the stator side of DFIG, $\mathrm{P}_{\mathrm{w}}$ is the total active power outputted by DFIG, and $\mathrm{Q}_{\mathrm{w}}$ is the total reactive power outputted by DFIG. Without giving consideration for copper and iron losses of rotor windings, the active power outputted by DFIG as shown in Fig. 1 shall meet:

$$
\left\{\begin{array}{l}
P_{w}=P_{s}+P_{g} \\
Q_{w}=Q_{s}+Q_{g}
\end{array}\right.
$$

Therein, $P_{g}=-s P_{s}$

If the direction of phase-A voltage of the power grid is consistent with d-axis, then $u_{\mathrm{gd}}=u_{\mathrm{g}}$ and $u_{\mathrm{gq}}=0$. The active and reactive currents outputted by DFIG to the power grid through the stator is:

$$
\left\{\begin{array}{l}
I_{s d}=\frac{L_{m}}{L_{s}} I_{r d} \\
I_{s q}=\frac{U_{g}}{L_{s} \omega_{s}}+\frac{L_{m}}{L_{s}} I_{r q}
\end{array}\right.
$$

Therein, ${ }^{\omega_{s}}$ is the angular spin rate of the stator, Ls is the leakage inductance of the stator and $\mathrm{Lm}$ is the exciting inductance.

Under normal power grid condition, the generators actively output according to maximum power tracking method and the grid-side converter operates under unit power factor mode, and DFIG system doesn't output reactive power. Actually, DFIG has certain reactive outputting capability. Stator-side active and reactive power operation range of DFIG is mainly restrained by the rotor-side converter:

$$
\sqrt{{I_{r d}}^{2}+I_{r q}^{2}} \leq I_{r, \max }
$$

Therein, $I_{r, \max }$ is the maximum allowable rotor-side current of the generator and is set as 1.5 times of the rated current.

Put formula (2) into formula (3) to obtain:

$\frac{U_{g}}{L_{s} \omega_{s}}-\sqrt{\left(\frac{L_{m}}{L_{s}} I_{r, \max }\right)^{2}-I_{s d}{ }^{2}} \leq I_{s q} \leq \frac{U_{g}}{L_{s} \omega_{s}}+\sqrt{\left(\frac{L_{m}}{L_{s}} I_{r, \max }\right)^{2}-I_{s d}{ }^{2}}$

Therefore, the maximum inductive reactive current able to be absorbed by the stator is:

$$
I_{s q, \mathrm{in}}^{\max }=\min \left\{\sqrt{\left(\frac{L_{m}}{L_{s}} I_{r, \max }\right)^{2}-I_{s d}{ }^{2}}-\frac{U_{g}}{L_{s} \omega_{s}}, I_{s, \max }\right\}
$$

Therein, $I_{\mathrm{s}, \max }$ is the maximum allowable stator-side current of the generator.

The limit range of the reactive power outputted by grid-side converter is:

$-I_{g, \text { max }}+I_{g d} \leq I_{g q} \leq I_{g, \max }-I_{g d}$

Therein, $I_{g, \max }$ is maximum allowable current of GSC. The reactive output capability of DFIG has provided the basis for HVRT. 


\section{Realization of high voltage ride-through of DFIG}

In order to realize the high voltage ride-through of DFIG and ensure that DFIG still can continuously run for more than $60 \mathrm{~ms}$ when the grid voltage swells to 1.3 times of the rated voltage, it is necessary to firstly enable the internal components of DFIG to meet relevant operating requirements during the operation and then adopt certain rules to coordinate and control the active and reactive powers outputted by the grid-side converter and the stator of DFIG.

The stable control of DC bus voltage amplitude is the mark for judging whether the wind power generators can realize high voltage ride-through. In order to ensure the stable control of the normal operation of GSC and DC voltage during the voltage swell of the power grid, DC bus voltage needs to meet:

$$
V_{d c} \geq \sqrt{3} \sqrt{\left(U_{g}+\omega_{r} L_{g} I_{g q}\right)^{2}+\left(\omega_{r} L_{g} I_{g d}\right)^{2}}
$$

Therein, $\omega_{r}$ is the angular spin rate of the rotor, $\mathrm{Lg}$ is the incoming inductance of the grid-side converter, $I_{g d}$ is the active current component outputted by grid-side converter, $I_{g q}$ is the reactive current component outputted by the grid-side converter.

The right side of formula (7) is expressed as $\mathrm{V}_{\mathrm{dc}}$, min. When GSC absorbs certain inductive reactive current, namely gq $<0$, then $\mathrm{V}_{\mathrm{dc} \text {,min }}$ is reduced. Therefore, when the voltage swell of the power grid causes the voltage rise of the wind power generator port, the normal operation of GSC can be realized through the control of $\mathrm{I}_{\mathrm{gq}}$. In order to ensure the normal operation of DC-side capacitor and the safe operation of RSC and GSC components, the control process shall meet:

$$
V_{d c}<k_{v} V_{d c}^{\max }
$$

Therein, $k_{v}$ is safety coefficient and is set as 0.95 in the article; $V_{d c}^{\max }$ is the maximum continuous operating voltage of DC-side capacitor.

The rational control of the reactive current outputted by GSC can enable DC-side voltage of the converter to be stabilized at a certain given value $V_{d c, R}$ able to meet formulae (7) and (8). When the voltage swell of the power grid causes the voltage of the wind power generator port to rise to $U_{g}^{\prime}$, in order to keep DC-side capacitance at a given voltage $\mathrm{V}_{\mathrm{dc}, \mathrm{R}}$, the minimum reactive current outputted by GSC is:

$$
I_{g q}=\frac{1}{\omega_{r} L_{g}}\left(\sqrt{\frac{V_{d c, R}{ }^{2}}{3}-\left(\omega_{r} L_{g} I_{g d}\right)^{2}}-U_{g}^{\prime}\right)
$$

When the port voltage of GSC is increased and GSC outputs rated reactive power to keep DC-side voltage at rated value, the port voltage at this moment is expressed as $U_{\mathrm{gsc}, \mathrm{R}}$. According to the parameters in the calculation examples in the article and formula (9), when the port voltage of SGC reaches $U_{\mathrm{gsc}, \mathrm{R}}=1.15$, the output current thereof is $0.3 p$.u. and GSC still can keep DC-side voltage at rated value. If $\mathrm{V}_{\mathrm{dc}, \mathrm{R}}=1100 \mathrm{~V}$ is kept, then $\mathrm{U}_{\mathrm{g}}$ cannot exceed 1.15; if $\mathrm{V}_{\mathrm{dc}, \mathrm{R}}=1187 \mathrm{~V}$ is kept, then $\mathrm{U}_{\mathrm{g}}$ cannot exceed 1.24; in other words, if $\mathrm{U}_{\mathrm{g}}<1.24$, then RSC and GSC can both keep normal operation. If the voltage continuously rises, then the output range of GSC will be exceeded. Therefore, only relying on the reactive power outputted by GSC cannot meet the high voltage ride-through requirement of wind power generators.

\section{Simulation research}

The simulation parameters are as follows: the radius of the blades of DFIG is $40 \mathrm{~m}$, the rated wind speed is $12 \mathrm{~m} / \mathrm{s}$, the cut-in wind speed is $6 \mathrm{~m} / \mathrm{s}$ and the cut-out wind speed is $15 \mathrm{~m} / \mathrm{s}$; generator parameters: the rate power is $2 \mathrm{MW}$, the stator-rotor ratio is 0.333 , the rated voltage of the stator is $690 \mathrm{~V}$, the resistance of the stator is $0.00489 \mathrm{pu}$, the resistance of the rotor is $0.0055 \mathrm{pu}$, the exciting inductance is $3.954 \mathrm{pu}$, the leakage inductance of the stator is $0.0924 \mathrm{pu}$ and the leakage inductance of the rotor is $0.0996 \mathrm{pu}$; converter parameters: the rated capacity of GSC is 600kVA, the rated capacity of RSC is $800 \mathrm{kVA}$, the rated voltage of DC bus is $1100 \mathrm{~V}$, the maximum continuous 
operating DC voltage is $1250 \mathrm{~V}$, the switching frequency of $\mathrm{PWM}$ is $2 \mathrm{kHz}$, the grid-side incoming inductance is $0.5 \mathrm{mH}$ and DC energy storage capacitance is $10 \mathrm{mF}$; transformer (T1) parameters: the rated capacity is $2.5 \mathrm{MWA}$ and the reactance is 0.1 p.u.

The wind speed of the wind power generator is $11 \mathrm{~m} / \mathrm{s}$, and the stable output thereof is active power 0.83 p.u. When the grid voltage swells to 1.12 times of the nominal valve at $t=4 \mathrm{~s}$, the fault lasts for $600 \mathrm{~ms}$ and the active power output of the wind power generator is not changed at this moment. The reactive powers outputted by GSC and generator stator are as shown in Fig. 2, and the corresponding DC-side bus voltage is as shown in Fig. 3, without Chopper circuit trigger. In Fig. 2, the reactive current outputted by GSC is zero, the stator-side reactive current is quantitative, DC-side bus voltage still can be kept at the rated value, the coordination control of the reactive output of GSC and at the generator stator-side can ensure the connected operation of the generator unit during voltage swell and meanwhile reduce the voltage at the stator side (grid side) of DFIG by $3 \%$, thus to facilitate the rapid recovery of the fault power grid and the safe operation of other interconnection loads.

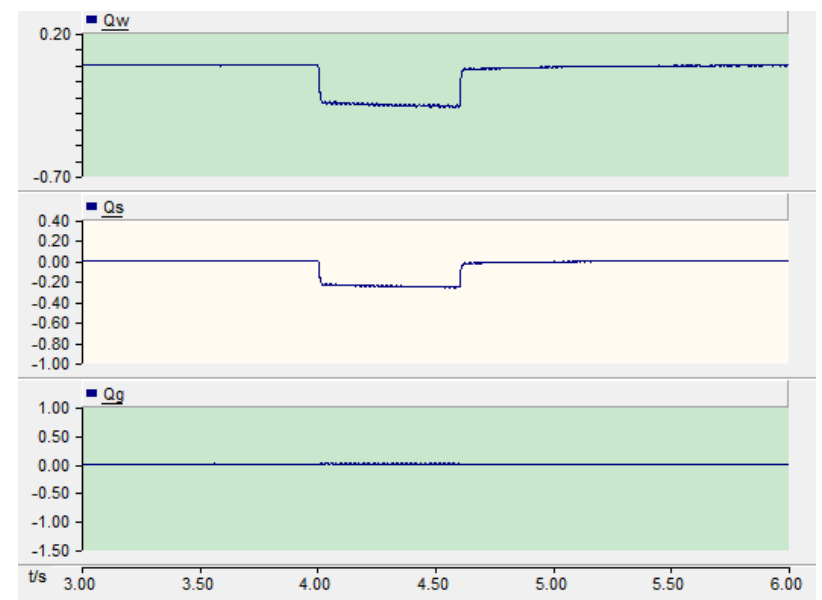

Fig. 2 The reactive power DFIG with the proposed scheme during grid voltage swell $\left(\mathrm{U}_{\mathrm{g}}=1.12\right)$

Under the same power grid condition, through the method proposed in the article, GSC rapidly injects inductive reactive power ${ }_{g q}$ into the fault power grid, RSC controls DFIG to preferentially output reactive power to the power grid so as to support the rapid recovery of the fault power grid. Under the condition of $\mathrm{Ug}, \mathrm{R}=1.2$, in order to keep the port voltage, the active power outputted at the generator stator side is zero, the wind power generator stator and GSC of DFIG output a lot of reactive power according to the maximum capacity, as shown in Fig. 3.

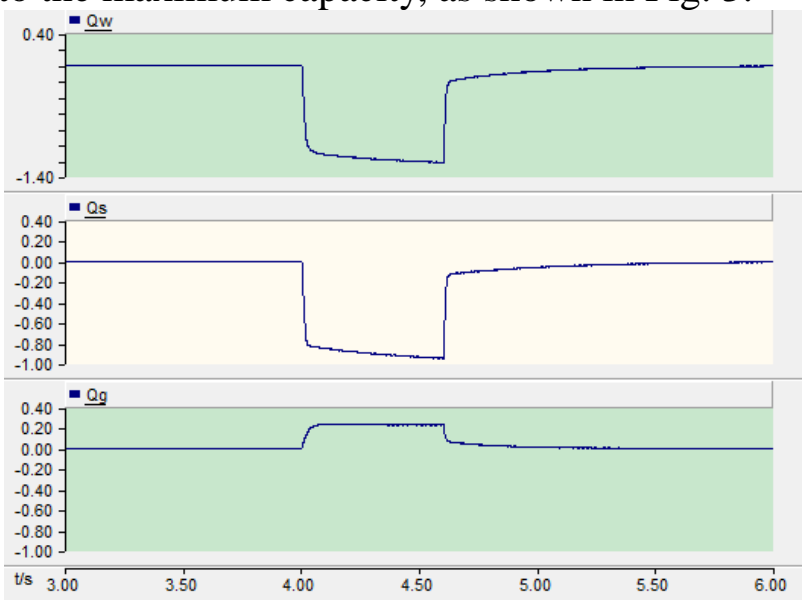

Fig. 3 The reactive power DFIG with the proposed scheme during grid voltage swell $\left(U_{g}=1.3\right)$

\section{Conclusion}

During grid voltage swell, the reactive outputs of the grid-side converter of DFIG and the generator stator are concertedly controlled so as to keep the safe and stable operation of DC-side bus voltage and accordingly realize the high voltage ride-through of the generator unit. The 
grid-side converter has weak reactive output capacity, so only relying on the reactive output thereof cannot meet the requirement for the safe operation of DC-side bus voltage and the reactive power outputted at the generator stator side shall be preferentially considered. Meanwhile, the high voltage ride-through capacity of DFIG is related to such factors as DC-side capacitor of the converter, incoming inductance and fault line (resistance) reactance. When the system voltage is very high, it is necessary to adopt active voltage control to restrain the port voltage of DFIG within the acceptable safe range of the wind power generator so as to realize the high voltage ride-through of the generator unit.

\section{References}

[1]Zhong, Chen, Stefan Müller Arisona, Xianfeng Huang, Michael Batty, and Gerhard Schmitt. "Detecting the dynamics of urban structure through spatial network analysis." International Journal of Geographical Information Science 28, no. 11 (2014): 2178-2199.

[2]Y. Geng, J. He, K. Pahlavan, Modeling the Effect of Human Body on TOA Based Indoor Human Tracking[J], International Journal of Wireless Information Networks 20(4), 306-317

[3]Zhang, Mengxin, Zhihan Lv, Xiaolei Zhang, Ge Chen, and Ke Zhang. "Research and Application of the 3D Virtual Community Based on WEBVR and RIA." Computer and Information Science 2, no. 1 (2009): p84.

[4]Jiang, Dingde, Zhengzheng Xu, Peng Zhang, and Ting Zhu. "A transform domain-based anomaly detection approach to network-wide traffic." Journal of Network and Computer Applications 40 (2014): 292-306.re

[5]Y. Geng, J. Chen, K. Pahlavan, Motion detection using RF signals for the first responder in emergency operations: A PHASER project[C], 2013 IEEE 24nd International Symposium on Personal Indoor and Mobile Radio Communications (PIMRC), London,Britain Sep. 2013 